\title{
M100 and NGC 6951: Morphological Clues to Central Dynamics
}

\section{J. H. Knapen}

Université de Montréal, Dép. de Physique, C.P. 6128, Succ. Centre Ville, Montréal, Québec, H3C 3J7 Canada

I. Shlosman

Dept. of Physics and Astronomy, Univ. of Kentucky, Lexington, KY 40506-0055, USA

J. E. Beckman

Inst. de Astrofísica de Canarias, E-38200 La Laguna, Tenerife, Spain

C. H. Heller

Univ. Sternwarte, Geismarlandstraße 11, 37083 Göttingen, Germany

R. F. Peletier

Kapteyn Inst., Postbus 800, NL-9700 AV Groningen, the Netherlands

R. S. de Jong

Univ. of Durham, Physics Dept., South Road, Durham DH1 3LE, UK

D. Nadeau and R. Doyon

Université de Montréal, Dép. de Physique, C.P. 6128, Succ. Centre Ville, Montréal, Québec, H3C 3J7 Canada

\section{Abstract.}

High-resolution optical and NIR observations are used to constrain a dynamical model of the circumnuclear star forming (SF) region in the barred galaxy M100 (NGC 4321). Small leading arms observed in our $K$ band image of the nuclear region have been reproduced in numerical modeling of M100, a galaxy with a double inner Lindblad resonance (ILR). We also present preliminary optical and NIR observations of NGC 6951: a barred galaxy with circumnuclear SF showing a distinctly different behavior to M100 at $2.2 \mu \mathrm{m}$.

\section{Introduction}

The main arguments in favor of multiple inner Lindblad resonances (ILRs) in the central regions of barred spirals, an idea that goes back to Sanders \& Huntley 
(1976) and even earlier, are the shape of the rotation curve, the shape of the dust lanes along the leading edges of the bars, and so-called nuclear rings of star formation (SF), possibly accompanied by molecular rings. Understanding the occurrence and behavior of gas streaming and SF near the ILRs is important since it can provide us with valuable checks on the dynamics in the central region of barred galaxies, and can possibly lead to a further understanding of the process of transporting gaseous material toward the nuclear regions of barred galaxies, and active galaxies in general.

Arsenault et al. (1988) concluded from the shape of the rotation curve that M100 (NGC 4321) has a double ILR. This galaxy has a moderately weak bar, and two distinct spiral arms that can be traced from the main disk, through the bar, into the inner $\mathrm{kpc}$. We have studied the central region in M100 in detail using optical and NIR imaging, and dynamical modeling (Knapen et al. 1995a, b). Our most important findings are discussed below. We also present some preliminary results for another galaxy - NGC 6951. This barred, late-type grand-design galaxy shows a similar structure to M100 in the optical, where a brightly star-forming circumnuclear region is obvious in e.g. $\mathrm{H} \alpha$ imaging. Kenney et al. (1992) found "twin peaks" of CO emission near the ring-like SF zone, which coincide with the main dust lanes.

\section{M100}

In blue light, and especially in $\mathrm{H} \alpha$, the central $2 \mathrm{kpc}$ region of M100 shows a set of tightly wound spiral armlets with massive SF. The arms can be traced outward through the bar of the galaxy and connect to the main spiral arm pair in the disk. They are accompanied by well-defined dust lanes, which are visible in Figure $1 \mathrm{~b}$ as a set of two dark features outlining the arm shape. In the NIR however the central region looks markedly different (see the $2.2 \mu \mathrm{m} \mathrm{K}$-image in Figure 1a). From inside out, the following components can be distinguished (see Knapen et al. 1995a): a small bulge component; an inner barlike region, with identical position angle and ellipticity to the large-scale bar; two leading arms; two symmetric peaks of emission; and the oval ring-like zone where the SF armlets are found in the optical. The $K$ contours in this zone are smooth and show hardly any indication of spiral arms or dust lanes. This change in morphology from optical to NIR is caused by different distributions of the stellar populations, along with the much reduced absorption by dust at $2.2 \mu$. The two symmetrically placed peaks of $K$ emission are massive starburst regions of roughly the same age (Knapen et al. 1995a), where at least part of the $K$ emission may come from young stars (e.g. O stars and $K$ supergiants). The fact that the $K$ isophotes become progressively elongated and skewed to the position angle of the bar both outside and inside the "ring" is a strong indicator in favor of a double ILR in this galaxy.

To understand the response of the gas to the underlying gravitational potential and especially the formation of the nuclear ring-like structure and associated features, we have modeled the stellar and gas dynamical processes there by means of $3 \mathrm{D}$ numerical simulations using a method described by Heller \& Shlosman (1994). Non-linear orbit analysis was used to verify positions of the 

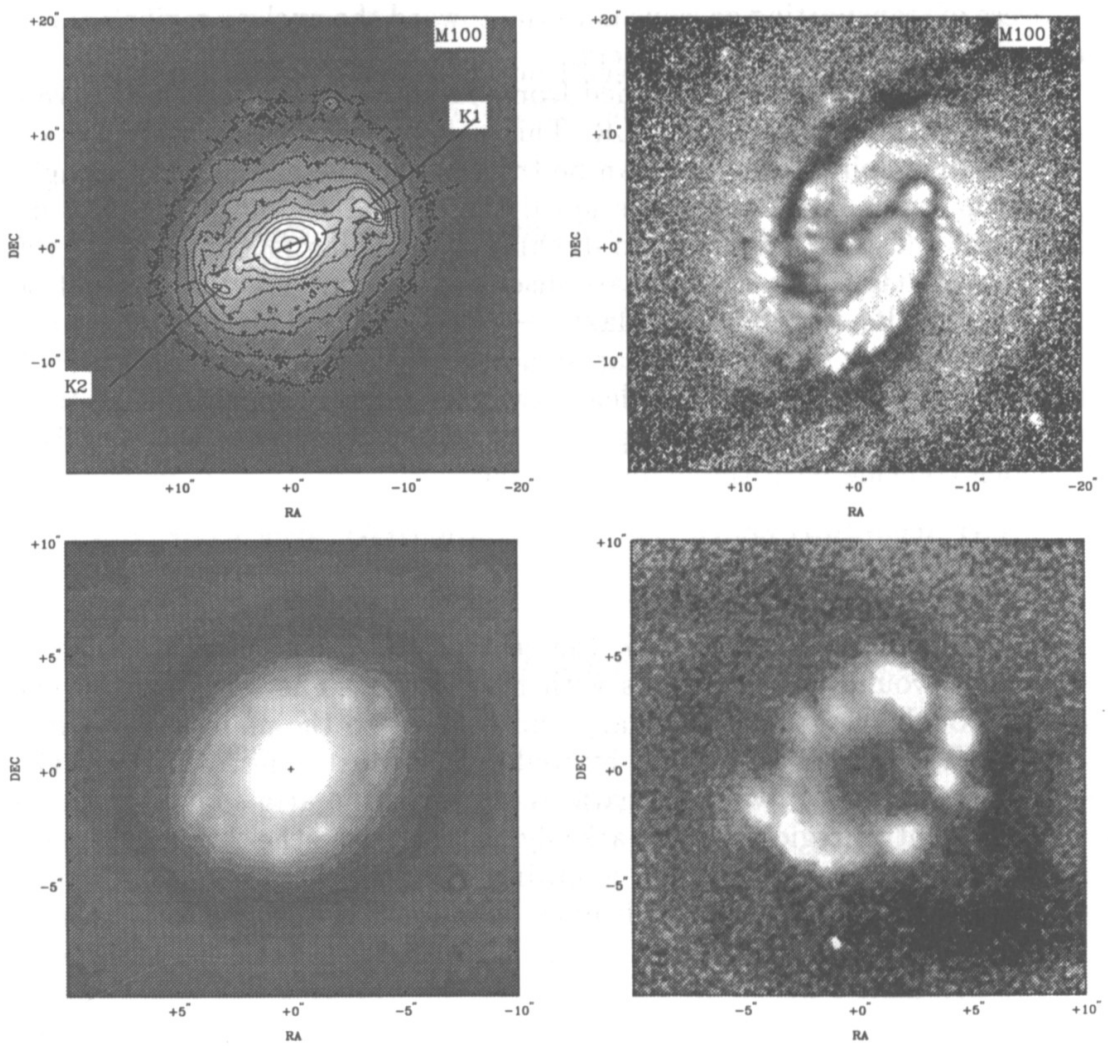

Figure 1. (a, upper left) $K$-band image of the central region of M100, as obtained on UKIRT. (b, upper right) $I-K$ color index image of the region of M100 shown in Figure 1a. Redder (more $K$ light) areas are darker, and indicate mostly dust lanes. (c, lower left) $K$-band image of the circumnuclear SF region of NGC 6951, as obtained with MONICA on the CFHT. (d, lower right) $B-I$ color index image of the same region of NGC 6951 as shown in Figure 1c. Bluer regions are represented as whiter in the figure. 
ILRs by determining the spatial extent of the family of orbits oriented along the minor axis of the bar.

Our modeling has shown explicitly that the dominant morphology in the center of M100 can be explained by the gas response to the stellar bar potential (Knapen et al. 1995b). We find that a system of trailing and leading shocks in the gas in the vicinity of the ILRs shows a robust behavior. We have been able to identify and model the main regions of SF there corresponding to four compression zones. Two zones of SF correspond to the so-called "twin peaks," and two additional ones are found where a pair of large-scale trailing shocks interacts with a pair of leading shocks. Dynamically and physically young massive stars $\left(\sim 10^{7} \mathrm{yrs}\right.$ and less) are present in the resonance region in addition to the old population. The young stars account for part of the $K$-emission and trace the gas flow.

\section{NGC 6951}

In Figure $1 c$ we show a $K$-band image (resolution $\sim 0.77$ ) of the central region of NGC 6951, as obtained with the MOntréal NIr CAmera (MONICA) on CFHT. Figure $1 \mathrm{~d}$ shows a $B-I$ color index image of similar resolution, made using images obtained at the $4.2 \mathrm{~m}$ WHT. The circumnuclear SF ring-like zone is well visible in the $B-I$ map, showing SF sites regularly spaced along the "ring". But whereas in M100 the $K$-contours are smooth in the region of the optical spiral arms, the separate SF sites do show up clearly in $K$ in NGC 6951. The most dramatic differences between M100 and NGC 6951, however, are the absence of both the inner barlike region and the twisting of K-band isophotes interior to the SF ring in NGC 6951.

It is unlikely that the observed differences can be explained by invoking the stellar population content. Rather the underlying dynamics of the innermost regions in these two galaxies differ fundamentally. Based on our numerical simulations of M100, the possible explanation lies in the differences in the mass distribution within the central kpc. In particular, the inner ILR in NGC 6951 may be positioned much closer to the nucleus, which would change the outcome of a number of gas dynamical processes there.

\section{References}

Arsenault, R., Boulesteix, J., Georgelin, Y., \& Roy, J.-R. 1988, A\&A, 200, 29

Heller, C. H. \& Shlosman, I. 1994, ApJ, 424, 84

Kenney, J. D. P., Wilson, C. D., Scoville, N. Z., Devereux, N. A., \& Young, J. S. 1992, ApJ, 395, L79

Knapen, J. H., Beckman, J. E., Shlosman, I., Peletier, R. F., Heller, C. H. \& de Jong, R. S. 1995a, ApJ, 443, L73

Knapen, J. H., Beckman, J. E., Heller, C. H., Shlosman, I., \& de Jong, R. S. $1995 \mathrm{~b}$, ApJ, 454, 623

Sanders, R. H. \& Huntley, J. M. 1976, ApJ, 209, 53 